\title{
Editorial \\ Architectures and Protocols for Wireless Sensor and Actuator Networks
}

\author{
Piergiuseppe Di Marco ${ }^{1, *(\mathbb{C}) \text { and Pangun Park }}{ }^{2}$ \\ 1 Department of Information Engineering, Computer Science and Mathematics, University of L'Aquila, \\ 67100 L'Aquila, Italy \\ 2 Department of Radio, Information, Communications Engineering, Chungnam National University, \\ Daejeon 34134, Korea; pgpark@cnu.ac.kr \\ * Correspondence: piergiuseppe.dimarco@univaq.it
}

Citation: Di Marco, P.; Park, P. Architectures and Protocols for Wireless Sensor and Actuator Networks. J. Sens. Actuator Netw. 2021, 10, 52. https://doi.org/ $10.3390 /$ jsan 10030052

Received: 17 July 2021

Accepted: 26 July 2021

Published: 30 July 2021

Publisher's Note: MDPI stays neutral with regard to jurisdictional claims in published maps and institutional affiliations.

Copyright: (C) 2021 by the authors. Licensee MDPI, Basel, Switzerland. This article is an open access article distributed under the terms and conditions of the Creative Commons Attribution (CC BY) license (https:/ / creativecommons.org/licenses/by/ $4.0 /)$.

\section{Introduction}

Recent advances in wireless networking, sensing, computing, and control are revolutionizing how physical systems interact with information and physical processes such as Cyber-Physical Systems (CPS), Internet of Things (IoT), and Tactile Internet. In particular, sensing, control, and actuation applications are converging to IoT, with billions of wireless devices deployed to interconnect people, objects, and control machines intelligently for industrial, commercial, and healthcare domains. These emerging applications heavily rely on Wireless Sensor and Actuator Networks (WSANs) enabling seamless integration of communication, control, and computation.

In Wireless Sensor and Actuator Networks (WSANs), sensor nodes attached to the physical plant sample and transmit measurements to a remote controller via a wireless channel; controllers compute control command based on the data and forward them to actuators in order to influence the dynamics of the plant. We can identify two separate logical network domains: data collection for sensors-to-controller network and data dissemination for controller-to-actuators network. Sensors can be transmit-only devices, with pre-determined scheduling for transmissions and aggressive duty-cycling. On the other hand, actuators need to be able to receive commands from the controller in a timely fashion. Besides, removing cables for the data communication of sensors and actuators motivates the removal of the power supply to these nodes to achieve full flexibility. Wireless network design needs to consider the strict reliability and delay constraints in order to guarantee the overall system performance. The data transmissions should be sufficiently reliable and deterministic with the latency on the order of seconds, or even milliseconds, depending on the time constraints of the physical system. Furthermore, even if it serves a single application, the data collection and data dissemination have typically competing requirements coexisting in the same deployment area. This scenario becomes even more complicated when multiple services and control loops coexist on the same infrastructure.

Standards organizations, such as the 3rd Generation Partnership Project (3GPP), the Bluetooth Special Interest Group (SIG), and the Institute of Electrical and Electronics Engineers (IEEE), are extensively working on wireless protocol enhancements to support WSAN applications. High reliability and resiliency are possibly obtained by redundant deployments, distributed computation capabilities, and synchronized protocol operations. Accurate and computationally affordable techniques for protocol modeling and optimization are then fundamental to guarantee the integration of large-scale WSANs with various potentially competing service requirements. In addition, energy efficiency in WSANs requires extremely simple and passive device architectures, including radio-frequency identification (RFID) tags, and protocols that require minimum overhead and simplified channel access mechanisms. Energy-efficient and secure operations, adaptability to heterogeneous and strict requirements, and interoperability represent challenging aspects that are not covered by traditional solutions. 
This Special Issue includes scientific contributions on WSANs and systems considering the co-design of protocol layers and architecture for various applications in the healthcare domain, industrial automation, environmental monitoring, logistics, and retails.

\section{Summary of Contributions}

The first contributions in this Special Issue consider applications in the healthcare domain. The COVID-19 pandemic has accelerated research on technological solutions to help monitor and contain the spread of infectious diseases and improve access to medical services. Critical facilities such as nursing homes and hospitals have been severely exposed to the pandemic, but the currently available short-range wireless technologies still face limits in proximity accuracy, battery lifetime, and privacy in those complex indoor environments. This is a representative WSAN case where the sensing infrastructure (for proximity monitoring) needs to be well integrated with the actuation infrastructure (for exposure notification) In [1], a framework for building a Bluetooth mesh standard-based architecture to support contact tracing for critical healthcare facilities is presented. The infrastructure relies on a hierarchical design and standardized protocols for exchanging proximity data instead of a typical peer-to-peer fashion using smartphone apps. Experimental campaigns and simulations are used to validate the proposed solution. Moreover, the paper suggests standardization of Bluetooth mesh models that include configurable parameters when the technology is used for contact tracing. The great advantage of using a standardized protocol solution such as Bluetooth mesh is that the facility manager is not forced to deploy a complete infrastructure dedicated only to contact tracing and proximity detection. However, it can reuse existing Bluetooth devices as proximity monitors and deploy Bluetooth mesh devices that can be used for other purposes beyond contact tracing needs.

An alternative solution in the context of intelligent healthcare systems can be implemented using the IEEE 802.15.6 standard. In [2], a framework for designing and optimizing multiple access contention protocols in the IEEE 802.15.6 standard is proposed. A wireless body-area sensor network is considered, with sensor nodes that collect vital data signs such as body temperature, electrocardiography, sugar level, $\mathrm{SpO}$, and blood pressure from the human body and send them to a hub or a coordinator via wireless channels. An efficient medium access control protocol is one of the most crucial components in such a body-area network to provide quality service and reliable communication. The use of heterogeneous user priorities allows for prioritized communication and is exploited in this paper to reduce frame collisions. This is a useful way to discriminate requirements for, e.g, data collection and data dissemination in the same network. To improve the reliability and energy efficiency, the proposed solution aims at reducing collisions among nodes that belong to the same users' priority by scaling the contention window for those nodes, and collisions among nodes of different users' priorities, by adjusting the carrier sensing time for those user priorities. The paper presents an analytical model and extensive simulations that show the high performance of the proposed collision avoidance mechanism. Indeed, the throughput and the node energy efficiency are improved by a factor of three and two times, respectively, with respect to the standard 802.15.6 protocol, due to a communication design that considers the application requirements in terms of user priority.

The authors of [3] look at a cross-layer design of communication and application aspects considering the synthesis of multi-loop controllers for heterogeneous Linear TimeInvariant (LTI) systems explicitly. Classic design approaches for sampling, scheduling, and control policies include either time-based or event-based metrics to perform appropriate actions in response to the changes of the parameters of interest. The proposed solution in [3] specifically focuses on the combination of a time-based metric, namely the Ageof-Information, and a threshold-based triggering function. The control performance is measured by Linear-Quadratic Gaussian (LQG) cost functions. The sampling and control units reside at the control system layer and are designed in a distributed fashion, i.e., they are locally installed in every control loop and generate decision variables for their corresponding local control systems. The scheduling unit resides at the network layer and arbitrates the channel access in a centralized fashion. Under some mild assumptions 
on the information structures of the policy makers, the paper shows that the cross-layer co-design problem can be opportunely decomposed so that the control law remains fixed for a variety of sampling and scheduling policies within the specified classes that satisfy those assumptions on their information structures. It is shown that the performance of the proposed scheduling design outperforms conventional random access resource scheduling.

The design of efficient communication protocols for wireless sensor and actuator networks does not only need to take into account requirements from the application but also from the underlying propagation environment. In [4], the challenges and solutions for underwater communication are surveyed and evaluated. Currently, underwater communication has become relevant in many practical applications like pollution monitoring, seismic monitoring, remote control in the offshore oil industry, marine life monitoring, data collection, natural disasters monitoring, discovering natural resources, underwater pipeline monitoring, etc. To transmit and relay the data reliably from the bottom of the sea to the sinks at the surface, multi-hop communication and redundant deployments need to be utilized and properly configured, and the various routing protocols that have been proposed and optimized for these applications are compared. It is shown that different protocols are suitable for different use cases and functions depending on the priority of the demands and the deploying environment. Finally, this work highlights the expected research efforts to improve routing protocols for wireless sensor and actuator networks for enhanced underwater monitoring and exploration.

As the last contribution to this Special Issue, [5] considers the impact of security constraints on the design of an RFID system for use in retail use cases. RFID tag counterfeiting is a critical security problem induced by replicating a tag by either cloning its hardware component or copying its software so that the reader or user would not be able to tell if this tag is genuine or replicated. Existing solutions to this problem do not account for a typical use case of reselling. The authors of [5] address the problem by proposing an integrated protocol that verifies the ownership and status of the item for sale and, in addition, enables the ownership transfer of the resold item. The approach is based on the use of warranty tags. A warranty tag is a second tag attached to the item or to the warranty card of the product and is required to be in possession of an owner attempting to resell the item outside of the store. The system set up is very similar to a classic counterfeit verification protocol, and in order to verify if a product is stolen or not, the authors employ a server that includes the details of the tagged object and the associated warranty card given to the buyer by the retailer when the item was first purchased. A detailed security analysis based on strand spaces is presented to show that the proposed protocol is secure, private, and robust against known attacks.

\section{Conclusions}

This Special Issue features five interesting and high-quality papers exploring different scientific and technological issues related to wireless sensor and actuator networks and their use for modern monitoring and control applications. The underlying common message is that these new scenarios and services require careful analysis and understanding of the cross-layer interactions in the protocol stack, specially when data collection (e.g., sensing) and data dissemination (e.g., actuation) need to coexist in the same wireless network. Application requirements in terms of reliability, user priority, stability, privacy, and security need to be properly included in the design of the architecture and the communication protocol. Depending on the application, standard solutions with configurable parameters may provide the right trade-off between the need for performance optimization and the re-usability of the solution.

We hope the readers will appreciate this Special Issue for enhancing their knowledge on architectures and protocols for WSANs, designed and optimized for modern and relevant use cases.

Funding: The work of Pangun Park was supported by the Basic Research Laboratory (BRL) of the National Research Foundation (NRF-2020R1A4A2002021) and Institute of Information \& Communi- 
cations Technology Planning \& Evaluation (IITP) under Grant No. 2020-0-00187 funded by the Korea government.

Conflicts of Interest: The authors declare no conflict of interest.

\section{References}

1. Di Marco, P.; Park, P.; Pratesi, M.; Santucci, F. A Bluetooth-Based Architecture for Contact Tracing in Healthcare Facilities. J. Sens. Actuator Netw. 2021, 10, 2. [CrossRef]

2. Adnan, M.; Sallabi, F.; Shuaib, K.; Abdul-Hafez, M. Performance Enhancement of IEEE 802.15.6 Using Collision Avoidance Technique. J. Sens. Actuator Netw. 2020, 9, 33. [CrossRef]

3. Mamduhi, M.H.; Champati, J.P.; Gross, J.; Johansson, K.H. Where Freshness Matters in the Control Loop: Mixed Age-ofInformation and Event-Based Co-Design for Multi-Loop Networked Control Systems. J. Sens. Actuator Netw. $2020,9,43$. [CrossRef]

4. Haque, K.F.; Kabir, K.H.; Abdelgawad, A. Advancement of Routing Protocols and Applications of Underwater Wireless Sensor Network (UWSN)—A Survey. J. Sens. Actuator Netw. 2020, 9, 19. [CrossRef]

5. Khalil, G.; Doss, R.; Chowdhury, M. An Extended Reselling Protocol for Existing Anti-Counterfeiting Schemes. J. Sens. Actuator Netw. 2021, 10, 12. [CrossRef] 